\title{
Constitutive modelling of fabric anisotropy in sand
}

\author{
Z.W. Gao \\ School of Engineering, University of Glasgow, Scotland, UK
}

J.D. Zhao \& X.S. Li

Department of Civil and Environmental Engineering, Hong Kong University of Science and Technology, Hong Kong SAR, China

Y.F. Dafalias

Department of Civil and Environmental Engineering, University of California, Davis, USA

Department of Mechanics, School of Applied Mathematical and Physical Sciences, National Technical University of Athens, Greece

\begin{abstract}
Fabric and its evolution have significant effect on the mechanical behaviour of granular materials. A three-dimensional anisotropic model for granular material is proposed with proper consideration of fabric evolution. An explicit expression for the yield function is proposed in terms of the invariants and joint invariants of the stress ratio and fabric tensors. The material fabric is assumed to evolve with plastic shear deformation in a manner that its principal axes tend to become co-directional with those of the loading direction and its magnitude approaches a critical state value at large deformation. A non-coaxial and associated flow rule in the deviatoric stress space is employed based on the yield function. The model is capable of characterizing the complex anisotropic behaviour of granular materials under monotonic loading with fixed principal stress directions and meanwhile gives reasonable explanation for the micromechanical mechanism for static liquefaction and noncoaxiality between the stress and plastic strain increment axes.
\end{abstract}

\section{INTRODUCTION}

Transversely anisotropic fabric structure is commonly observed in both natural and manmade sand deposits and profoundly influences the mechanical behaviour of these soils including strength and dilatancy (Yoshimine et al. 1998, Gao et al. 2010). Proper consideration of the effect of fabric is important for safe design and maintenance of relevant key infrastructures (Uthayakumar \& Vaid 1998).

There have been many attempts on theoretical characterization and modelling of fabric anisotropy in sand and its effect on macroscopic sand behaviour. Among many, those models based on the use of rotated yield and plastic potential surfaces have gained limited popularity in the literature (Sekiguchi \& Ohta 1977, Pestana \& Whittle 1999). However, yield surface rotation may not be able to account for the anisotropic nature of sand related to particle orientation, contact normal and void space distribution properly, as the magnitude and direction of rotation is typically associated with the initial stress state (Kaliakin 2003). The employment of fabric tensors derived from the microstructural information of sand has proved to be efficient and physically more realistic in modelling sand behaviour (Oda \& Nakayama 1989, Pietruszczak 1999, Li \& Dafalias 2002, Dafalias et al. 2004). Being successful to a certain extent, these studies have commonly ignored the change of the fabric anisotropy during the deformation of the material, which is at odd with both ex- perimental and numerical observations, as the sand fabric will adjust to sustain the external loading in an optimum manner when it is deformed ( $\mathrm{Li} \& \mathrm{Li} 2009$, Zhao \& Guo 2013; Guo \& Zhao, 2013).

This paper presents an anisotropic sand model accounting for fabric evolution based on the anisotropic critical state theory (ACST) by Li \& Dafalias (2012). The new model features an explicit yield function expressed in terms of the invariants and joint invariants of the stress ratio tensor $r_{\mathrm{ij}}$ and a deviatoric fabric tensor $F_{\mathrm{ij}}$. Over a typical monotonic loading course, the fabric tensor is assumed to evolve towards the direction of loading. Based on the proposed framework, a non-coaxial flow rule is readily derived.

\section{CONSTITUTIVE MODEL}

\subsection{Yield function}

Based on this micromechanical deformation mechanism that the shear resistance of sand is contributed by inter-particle friction and fabric anisotropy, we propose the following yield function,

$$
f=\frac{R}{g(\theta)}-H e^{-k_{h}(A-1)^{2}}=0
$$

where $R=\left(3 r_{\mathrm{ij}} r_{\mathrm{ij}}\right)^{0.5}$ with $r_{\mathrm{ij}}=\left(\sigma_{\mathrm{ij}}-p \delta_{\mathrm{ij}}\right) / 3$ being the stress ratio tensor, in which $\sigma_{\mathrm{ij}}$ is the stress tensor, $p$ $=\sigma_{\mathrm{ij}} / 3$ is the mean normal stress, $\delta_{\mathrm{ij}}$ is the Kronecker delta; $H$ is a hardening parameter whose evolution 
law depends on the stress as well as internal variables including soil density and fabric; $A$ is a fabric anisotropy variable; $k_{\mathrm{h}}$ is a non-negative model constant with default value of $0.03 ; g(\theta)$ is an interpolation function based on the Lode angle $\theta$ of $r_{\mathrm{ij}}$ as follows (personal communication, Z.L. Wang 1992)

$$
g(\theta)=\frac{\sqrt{\left(1+c^{2}\right)^{2}+4 c\left(1-c^{2}\right) \sin 3 \theta}-\left(1+c^{2}\right)}{2(1-c) \sin 3 \theta}
$$

where $c=M_{\mathrm{e}} / M_{\mathrm{c}}$ is the ratio between the critical state stress ratio in triaxial extension $M_{\mathrm{e}}$ and that in triaxial compression $M_{\mathrm{c}}$.

An important inclusion in the yield function in Equation (1) is a fabric anisotropy variable $A$ that is defined by the following joint invariant of $F_{\mathrm{ij}}$ and $n_{\mathrm{ij}}$ (Li \& Dafalias 2004, Gao et al. 2014)

$A=F_{i j} n_{i j}$

where $F_{\mathrm{ij}}$ is a symmetric traceless tensor whose nor $\mathrm{m} F=\left(F_{\mathrm{ij}} F_{\mathrm{ij}}\right)^{0.5}$ is referred to as the degree of fabric a nisotropy. For convenience, $F_{\mathrm{ij}}$ is normalized such th at in critical state, $F$ is unity. The deviatoric unit loa ding direction tensor $n_{\mathrm{ij}}$ in Equation (3) is defined as follows (Li \& Dafalias 2004)

$$
n_{i j}=\frac{N_{i j}-N_{m n} \delta_{m n} \delta_{i j} / 3}{\left\|N_{i j}-N_{m n} \delta_{m n} \delta_{i j} / 3\right\|}
$$

with

$$
N_{i j}=\frac{\partial[R / g(\theta)]}{\partial R} \frac{\partial R}{\partial r_{i j}}+\frac{\partial[R / g(\theta)]}{\partial g(\theta)} \frac{\partial g(\theta)}{\partial \theta} \frac{\partial \theta}{\partial r_{i j}}
$$

Obviously, $n_{\mathrm{ii}}=0$ and $n_{\mathrm{ij}} n_{\mathrm{ij}}=1$. Notice that the $n_{\mathrm{ij}}$ is the deviatoric unit normal to a yield surface resulting from Equation (1) with the assumption that $A$ is a constant (in other words $n_{\mathrm{ij}}$ is not normal to the yield surface of Eq. (1)).

\subsection{Evolution law for $H$ and $F_{i j}$}

Within the hypothesis that sand's stress-strain response is incrementally linear, the evolution of the two internal variables is assumed to be

$$
\begin{aligned}
& d H=\langle L\rangle \frac{G\left(1-c_{h} e\right)}{p R}\left[M_{c} g(\theta) \mathrm{e}^{-n \zeta}-R\right] \\
& d F_{i j}=\langle L\rangle \Theta_{i j}=\langle L\rangle k_{f}\left(n_{i j}-F_{i j}\right)
\end{aligned}
$$

where $c_{h, n}$ and $k_{f}$ are three model parameters, $e$ is the current void ratio, \langle\rangle are the Macauley brackets such that $\langle L\rangle=L$ for $L>0$ and $\langle L\rangle=0$ for $L \leq 0, \zeta$ is the dilatancy state parameter defined as below (Li \& Dafalias 2012)

$$
\zeta=\psi-e_{A}(A-1)
$$

where $e_{A}$ is a model parameter, $\psi=e-e_{c}$ is the state parameter defined by Been \& Jefferies (1985) with $e_{c}$ being the critical state void ratio corresponding to the current $p$. In the present work, the critical state line in the $e-p$ plane is given by (Li \& Wang 1998) $e_{c}=e_{r}-\lambda_{c}\left(p / p_{a}\right)^{0.7}$

where $e_{\mathrm{r}}$ and $\lambda_{\mathrm{c}}$ are two material constants and $p_{\mathrm{a}}$ $(=101 \mathrm{kPa})$ is the atmospheric pressure. The above evolution law of $F_{\text {ij }}$ with plastic deformation expressed by Equation (7) leads towards coaxiality with the loading direction $n_{\mathrm{ij}}$.

\subsection{Dilatancy relation and flow rule}

A proper dilatancy relation $D$ defined as below is essential for modelling the sand behaviour

$$
D=\frac{d \varepsilon_{i i}^{p}}{\sqrt{2 d e_{i j}^{p} d e_{i j}^{p} / 3}}
$$

where $d \varepsilon_{i j}^{p}$ is the plastic strain increment and $d e_{i j}^{p}$ is the plastic shear strain increment. Based on $\mathrm{Li} \&$ Dafalias (2012), the following dilatancy relation which accounts for the effect of density, confining pressure and anisotropy is proposed

$$
D=\frac{d_{1}\left[1+R / M_{c} g(\theta)\right]}{M_{c} g(\theta)}\left[M_{c} g(\theta) e^{m \zeta}-R\right]
$$

where $d_{1}$ and $m$ are two model constants.

By assuming an associated flow rule in the deviatoric stress space based on the yield function in Equation (1), the increment of the plastic shear strain $d e_{i j}^{p}$ is expressed as

$d e_{i j}^{p}=\langle L\rangle m_{i j}$

where

$m_{i j}=\frac{\partial f / \partial r_{i j}-\left(\partial f / \partial r_{m n}\right) \delta_{m n} \delta_{i j} / 3}{\left\|\partial f / \partial r_{i j}-\left(\partial f / \partial r_{m n}\right) \delta_{m n} \delta_{i j} / 3\right\|}$

Notice that $m_{\mathrm{ij}}$ is normal to the yield surface expressed by Equation (1). Since $a f l a r_{\mathrm{ij}}$ consists of two parts with one being coaxial with $r_{\mathrm{ij}}$ (or equivalently $\sigma_{\mathrm{ij}}$ itself) and the other involving $F_{\mathrm{ij}}$ which is attributed to fabric anisotropy and is in general noncoaxial with $r_{\mathrm{ij}}$ (Gao et al. 2014), the flow rule expressed by Equations (12) and (13) naturally address the non-coaxiality issue in soil modelling.

\subsection{Elastic moduli}

As plastic strain dominates sand deformation, the influence of elastic anisotropy, if any, is considered negligible. The following elastic moduli (Richart et al. 1970, Li \& Dafalias 2012, Gao et al. 2014) are employed:

$$
\begin{gathered}
G=G_{0} \frac{(2.97-e)^{2}}{1+e} \sqrt{p p_{a}} \\
K=G \frac{2(1+v)}{3(1-2 v)}
\end{gathered}
$$


where $G$ and $K$ denote the elastic shear and bulk modulus, respectively, $G_{0}$ is a material constant and $v$ is the Poisson's ratio assumed to be a constant.

\section{MODEL SIMULATION FOR ANISOTROPIC SAND BEHAVIOUR}

\subsection{Model parameters}

To verify the model capability in simulating the anisotropic sand behaviour, we employ the test data for the dry-deposited Toyoura sand reported by Yoshimine et al. (1998). The model parameters are listed in Table 1 and the initial degree of anisotropy $F_{0}$ is set to be 0.45 . The procedure for parameter determination is discussed in Gao et al. (2014).

Table 1 Model parameters for Toyoura sand

\begin{tabular}{cc}
\hline Parameter & value \\
\hline$G_{0}$ & 125 \\
$v$ & 0.1 \\
$M_{\mathrm{c}}$ & 1.25 \\
$c$ & 0.75 \\
$e_{\mathrm{r}}$ & 0.934 \\
$\lambda_{\mathrm{c}}$ & 0.02 \\
$c_{\mathrm{h}}$ & 0.90 \\
$n$ & 3.0 \\
$d_{1}$ & 0.2 \\
$m$ & 5.3 \\
$e_{\mathrm{A}}$ & 0.10 \\
$k_{\mathrm{f}}$ & 5.7 \\
\hline
\end{tabular}

\subsection{Model simulation}

Figure 1 shows the model simulations for the anisotropic sand behaviour under undrained torsional loading with constant intermediate principal stress vatiable $b=0.25$. In this figure, $\alpha$ is the major principal stress direction with respect to the deposition direction and $D_{r c}$ is the relative density after consolidation. Clearly, the model well captures the trend that larger value of $\alpha$ generally leads to softer (lower shear stress $\sigma_{1}-\sigma_{3}$ at the same deviatoric strain $\left.\varepsilon_{1}-\varepsilon_{3}\right)$ and relatively more contractive sand response. Good agreement between the test data and model simulations can be observed.

An important feature of the present model is the non-coaxial flow rule in Equations (12) and (13), resulting naturally by the introduction of an evolving fabric tensor into the yield function and the associative flow rule assumption in the deviatoric stress space.

In a torsional shear test, the radial stress $\sigma_{\mathrm{r}}$ is always the intermediate principal stress and the radial strain $\varepsilon_{r}$ the intermediate principal strain. In this setting, it is convenient to use the model to explain the non-coaxiality in the $z-\theta$ plane (Gao et al. 2014). To elaborate on this point and motivated by the approach in Dafalias et al. (2004), we plot in Figure 2 the variation with deviatoric strain of the difference of the angle $\alpha(\sigma)$ between the direction of the major principal stress $\sigma_{1}$ and the vertical direction, from the angle $\alpha(\varepsilon)$ that the major principal strain $\varepsilon_{1}$ forms with the vertical direction. Such difference is a measure of non-coaxiality. The simulations match the experimental observation on non-coaxiality qualitatively well. When $\alpha=0^{\circ}$ or $90^{\circ}$, there is only change of the principal values of fabric tensor during the development of plastic strain, but no fabric rotation is involved. As such, the two sources of plastic strain increment due to stress and fabric increments will influence its value only, with its direction aligning with the stress direction during the entire loading course. Thus, the predicted sand response is generally coaxial, which is consistent with the experimental observation (Yoshimine et al. 1998). In all the other cases when $\alpha$ is between $0^{\circ}$ and $90^{\circ}$, coaxiality is assumed for purely elastic stage (below $0.5 \%$ deviatoric strain) due to the employment of isotropic elastic relation. Beyond this elastic stage to a relative low strain level (such as $2 \%$ ), however, a distinct difference between $\alpha(\varepsilon)$ and $\alpha(\sigma)$ of the order of 4 to 5 degrees on the average is found (Fig. 2), which indicates clearly non-coaxiality. Upon further loading, the fabric tends to rotate towards the direction of stress, and the difference between $\alpha(\varepsilon)$ and $\alpha(\sigma)$ predicted by the model decreases after the peak, and the non-coaxiality will totally disappear at large strain levels.

Figure 3 shows the model simulation for the sand fabric evolution in undrained triaxial extension where static liquefaction occurs. As the fabric tensor is initially triaxial-compression like due to the sample's method of preparation and coaxial with the loading direction, thus, it undergoes only a change of its norm, without any change of its principal directions. In particular the value of its major principal component decreases while the value of its minor principal component increases, which makes the norm $F$ undergo a decrease first until at $7 \%$ deviatoric strain. At this point, all components of the fabric tensor are 0 so that a transient isotropic state is observed $(F=A=0)$. As the deformation continues, the original minor component becomes the major one, whilst the original major one turns to be the minor one. The overall degree of anisotropy $F$ shows a slight rebound from zero (Fig. 3(c)). The anisotropic variable $A$ increases monotonically from a negative value through zero to a positive one (Fig. 3(c)). Nevertheless, both $A$ and $F$ reach a very small positive value at static liquefaction where $p=0$, which is far smaller than their respective critical state value had liquefaction not occurred, which is also observed in the DEM simulations by Li and Li (2009). 


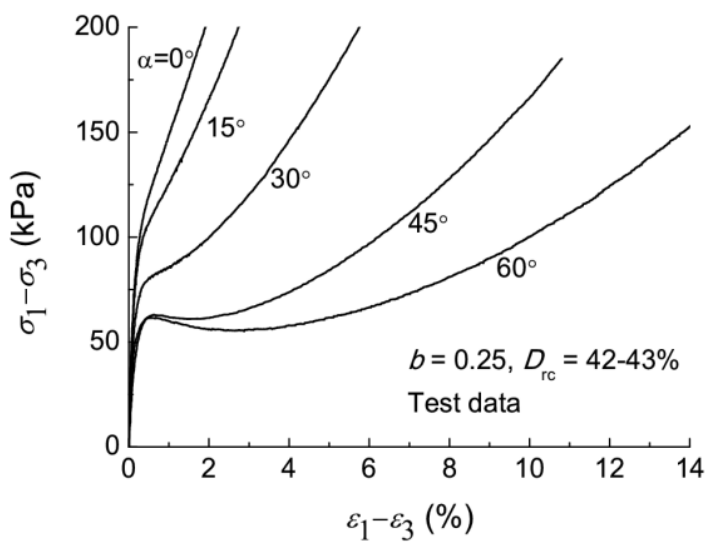

(a)

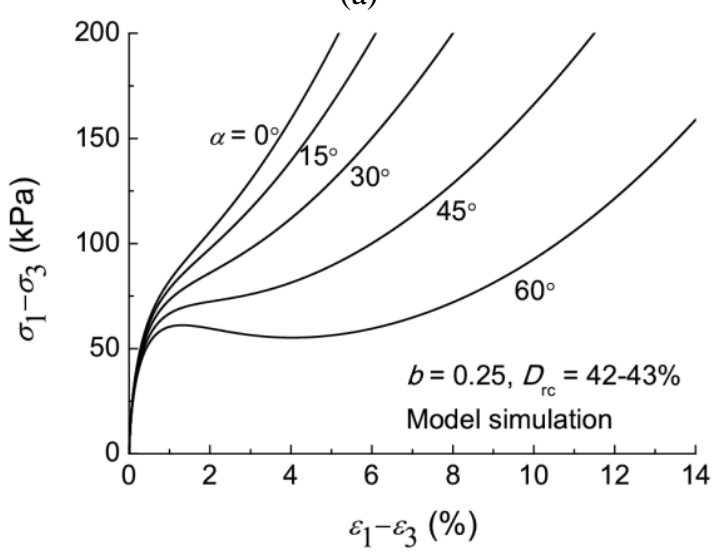

(b)

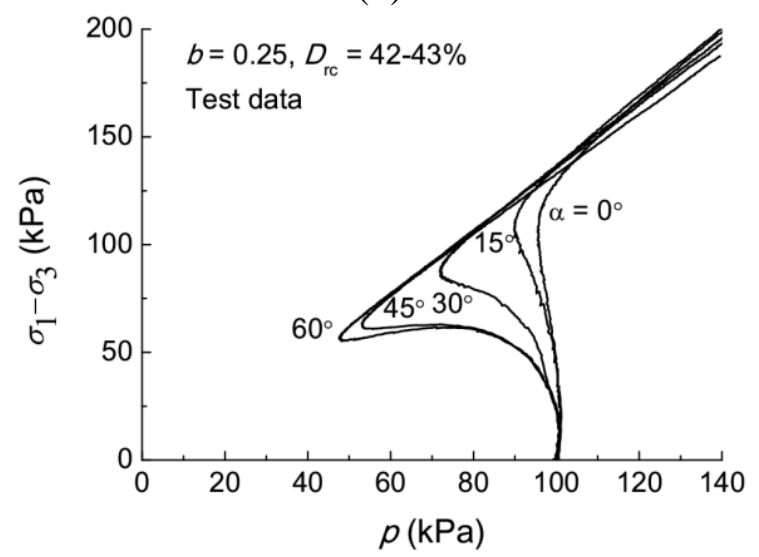

(c)

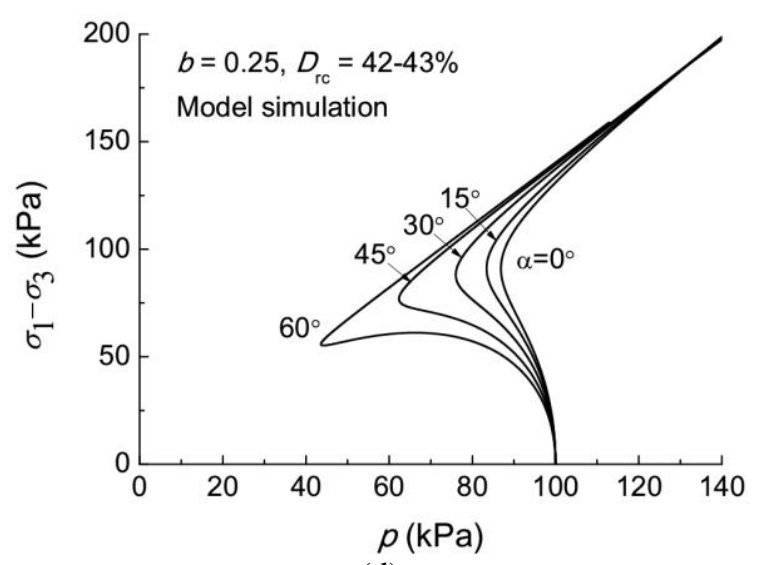

(d)

Figure 1. Model simulation for the anisotropic sand behaviour in undrained torsional shear tests (data from Yoshimine et al. 1998).

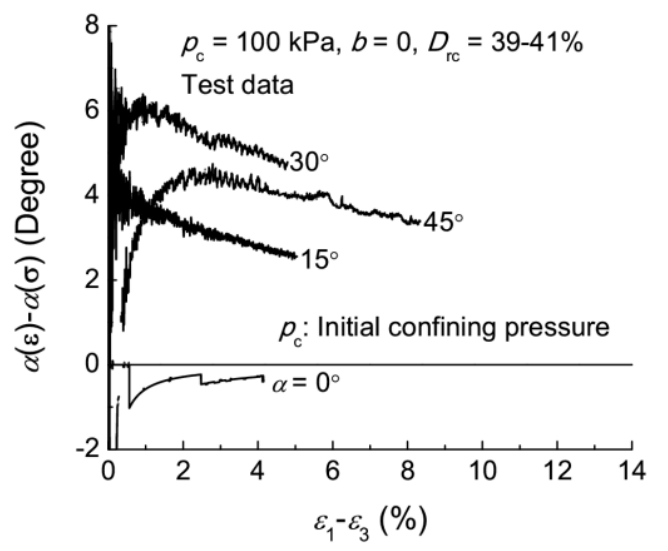

(a)

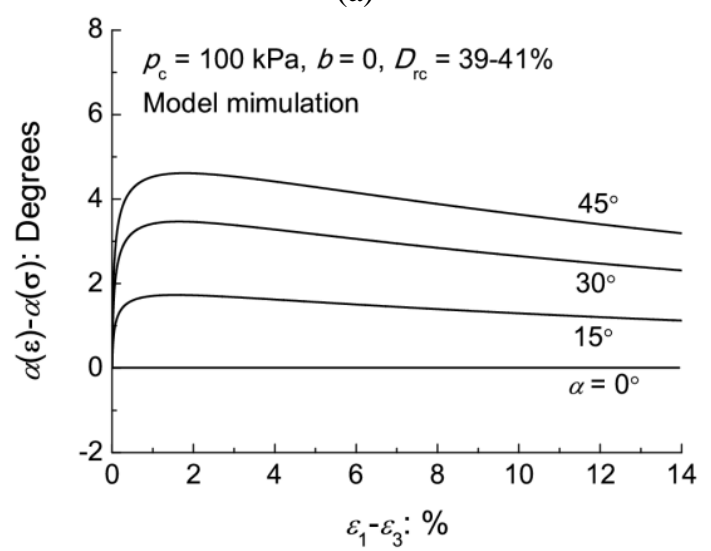

(b)

Figure 2. Comparison between the observed and model predicted non-coaxial sand behaviour in undrained torsional shear tests (data from Yoshimine et al. 1998).

\section{CONCLUSIONS}

A three-dimensional elasto-plastic constitutive model has been proposed to describe the anisotropic behaviour of sand under monotonic loading with fixed principal stress directions. The model constructed within the framework of ACST recently presented by $\mathrm{Li}$ and Dafalias (2012), which emphasizes the role of fabric on the characterization of sand response at critical state. The model employs a voidbased fabric tensor and a physically-based fabric evolution law to account for the influence of void size and orientation and their change during shear on the sand behaviour including plastic hardening and dilatancy. At the critical state, the fabric tensor has a constant magnitude and is co-directional with the loading direction. A non-coaxial but associative flow rule in the deviatoric stress space is used and it can naturally account for the non-coaxial behaviour of initially anisotropic sand samples under monotonic loading.

The model has been used to simulate the undrained test results for the dry-deposited Toyoura sand (Yoshimine et al., 1998) under undrained torsional shear tests with fixed principal stress direction and constant intermediate principal stress variable. The model simulations compare well with the test results. 


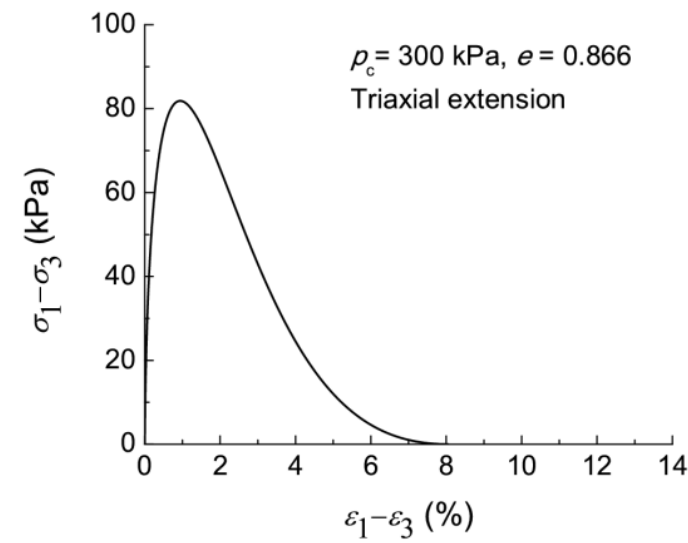

(a)

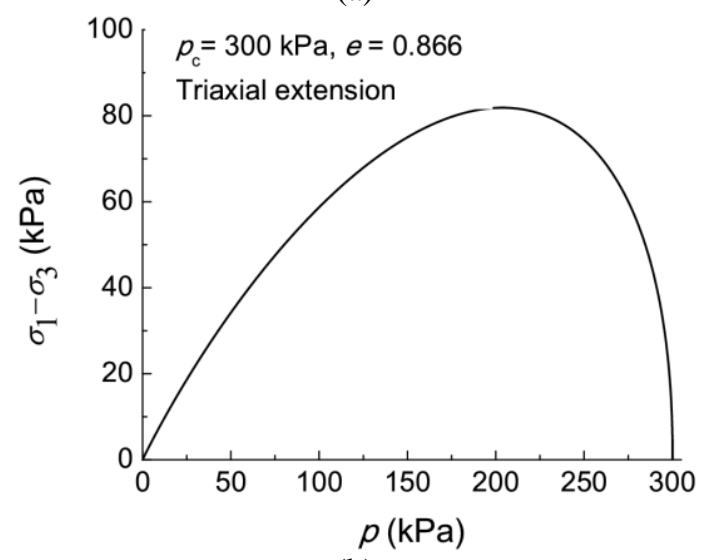

(b)

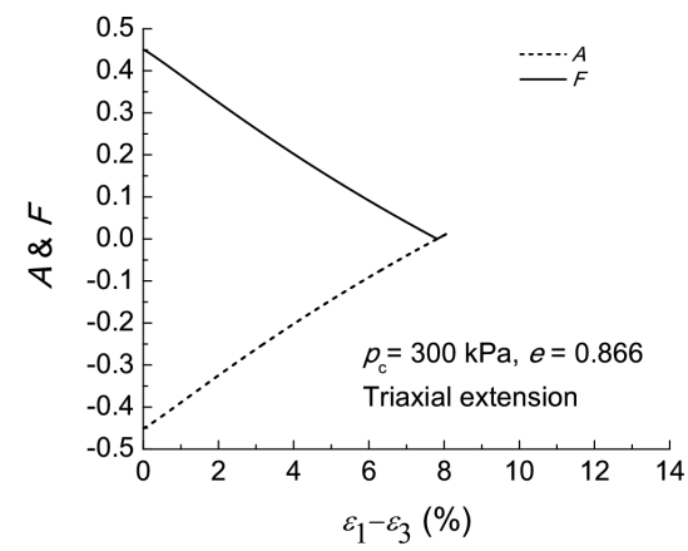

(c)

Figure 3. Model simulation for the sand behaviour in undrained triaxial extension and the fabric evolution (static liquefaction occurs).

\section{ACKNOWLEDGEMENT}

Y.F. Dafalias would like to acknowledge support from the European Research Council under the European Union's Seventh Framework Program FP7ERC-IDEAS Advanced Grant Agreement n 290963 (SOMEF), and partial support by NSF project CMMI-1162096.

\section{REFERENCES}

Been, K. \& Jefferies, M.G. 1985. A state parameter for sands. Géotechnique 35(2): 99-112.
Dafalias, Y.F., Papadimitriou, A.G. \& Li, X.S. 2004. Sand plasticity model accounting for inherent fabric anisotropy. Journal of Engineering Mechanics 130(11): 1319-1333.

Gao, Z.W., Zhao, J.D. \& Yao, Y.P. 2010. A generalized anisotropic failure criterion for geomaterials. International Journal of Solids and Structures 47(22-23): 3166-3185.

Gao, Z.W., Zhao, J.D., Li, X.S. \& Dafalias, Y.F. 2014. A critical state sand plasticity model accounting for fabric evolution. International Journal for Numerical and Analytical Methods in Geomechanics 38(4): 370-390.

Guo, N. \& Zhao, J.D. 2013. The signature of shear-induced anisotropy in granular media. Computers and Geotechnics 47: $1-15$.

Kaliakin, V.N. 2003. An assessment of the macroscopic quantification of anisotropy in cohesive soils. Prco. 1st JapanU.S. Workshop on Testing, Modeling, and Simulation, Boston, Massachusetts, USA, 370-393.

Li, X.S. \& Dafalias, Y.F. 2002. Constitutive modelling of inherently anisotropic sand behaviour. Journal of Geotechnical and Geoenvironmental Engineering 128(10): 868880.

Li, X.S. \& Dafalias, Y.F. 2004. A constitutive framework for anisotropic sand including non-proportional loading. Géotechnique 54(1): 41-55.

Li, X.S. \& Dafalias, Y.F. 2012. Anisotropic critical state theory: the role of fabric. Journal of Engineering Mechanics 138(3): 263-275.

Li, X.S. \& Li, X. 2009. Micro-Macro quantification of the internal structure of granular materials. Journal of Engineering Mechanics 135(7): 641-656.

Li, X.S. \& Wang, Y. 1998. Linear representation of steadystate line for sand. Journal of Geotechnical and Geoenvironmental Engineering 124(12): 1215-1217.

Oda, M. \& Nakayama, H. 1989. Yield function for soil with anisotropic fabric. Journal of Engineering Mechanics 115(1): 89-104.

Pestana, J.M. \& Whittle, A.J. 1999. Formulation of a unified constitutive model for clays and sands. International Journal for Numerical and Analytical Methods in Geomechanics 23(12): 1215-1243.

Pietruszczak, S. 1999. On inelastic behaviour of anisotropic frictional materials. Mechanics of Cohesive-Frictional Materials 4(3): 281-293.

Richart, F.E. Jr., Hall, J.R. \& Woods, R.D. 1970. Vibrations of soils and foundations. Englewood Cliffs, NJ: Prentice-Hall.

Sekiguchi, H. \& Ohta, K. 1977. Induced anisotropy and time dependency in clays. Constitutive Equations of Soils, Proceedings of the 9th International Conference on Soil Mech. Found. Eng., Special Session 9, Tokyo, 229-238.

Uthayakumar, M. \& Vaid, Y.P. 1998. Static liquefaction of sands under multiaxial loading. Canadian Geotechnical Journal 35(2): 273-283

Yoshimine, M., Ishihara, K. \& Vargas, W. 1998. Effects of principal stress direction and intermediate principal stress on undrained shear behaviour of sand. Soils Foundations 38(3): 179-188.

Zhao, J.D. \& Guo, N. 2013. Unique critical state characteristics in granular media considering fabric anisotropy. Géotechnique 63(8): 695-704. 